RHIC-PH-13

Strangeness Evolution in the Central Region of a Heavy Ion Collision with Transverse Flow Effects

K. Kajantie, M. Kataja, P.V. Ruuskanen

Brookhaven National Laboratory

August 1986 
DEPARTMENT OF PHYSICS, UNIVERSITY OF JYVÄSKYLÄ PREPRINT No. 9/1986

Strangeness evolution in the central region of a heavy ion collision with transverse flow effects

K. Kajantie, M. Kataja and P. V. Ruuskanen

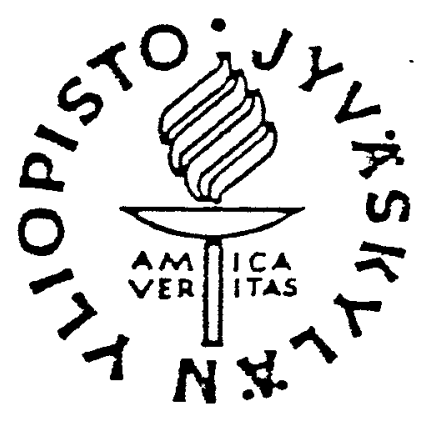

DEPARTMENT OF PHYSICS .

UNIVERSITY OF JYVÄSKYLÄ

Seminaarinkatu 15

SF-40100 JYVÄSKYL $\ddot{A} 10$

FINLAND 


\title{
Strangeness evolution in the central region of a heavy ion collision with transverse flow effects
}

\author{
K. Kajantie ${ }^{1}$, M. Kataja ${ }^{2}$ and P. V. Ruuskanen ${ }^{2}$
}

\begin{abstract}
Strangeness evolution in the central region of an ultra-relativistic nucleus-nucleus collision is studied by using a numerically computed boost invariant cylindrically symmetric hydrodynamic flow. Kinetic equations describing the evolution through quark-gluon plasma, mixed and hadron phases are derived. With reasonable collision terms the conclusion is that the strangeness density (=weighted sum of the strange quark density and the sum of $K^{-}$and $\bar{K}^{\circ}$ densities) evolves close to or up to a factor 2 below the equilibrium density until the end of the mixed phase. With transverse expansion the dilution of matter in hadron phase is so fast that strangeness creation and annihilation processes effectively decouple. This implies that the observed $N_{K}-/ N_{\pi^{-}}$is rather independent of the assumed decoupling temperature and lies in the range $0.15-0.3$.
\end{abstract}

1) Academy of Finland and Department of Theoretical Physics, Siltavuorenpenger $20 \mathrm{C}$, SF-00170 Helsinki, Finland.

2) Department of Physics, Seminaarinkatu 15, SF-40100 Jyväskylä,Finland. 
A large relative strangeness abundance is often discussed as a signal for quark-gluon plasma [1-2]. Consider the baryon number-free central region, which probably is simpler than the baryon-rich fragmentation regions. For $T_{i} \gg m_{a}$ (=150 MeV, for definiteness) one clearly must have $n_{u}=n_{\bar{u}}=n_{d}=n_{\bar{d}} \approx n_{s}=n_{\bar{s}}$, initially. To correctly assess the relative amount of strangeness, it is essential to include gluons and consider the ratio $n_{s} / s$, $s=$ entropy density. Furthermore, since the constant total entropy $S$ of the event can be measured by observing the multiplicity $N_{\text {tot }}=S / c, c=3.6$ (neglecting mass effects in the hadron gas), an appropriate measure of the initial abundance is

$$
\begin{aligned}
\frac{n_{s}}{n_{\text {tot }}} \approx \frac{3.6 n_{s}}{s} & =0.095 & & N_{F}=2 \\
& =0.12 & & N_{F}=3,
\end{aligned}
$$

using ideal gas formulas. This is not very large. However, the experiments only observe the final strangeness and the question now is what happens to this initial abundance in the course of the hydrodynamic expansion.

The behaviour of strangeness under scaling hydrodynamic flow of baryon-free QCD matter with a first order equation of state has been discussed in [3] neglecting transverse flow. The aim of this letter is to derive kinetic equations describing the evolution of strangess through quark-gluon plasma, mixed and hadron phases for a general three-dimensional expansion of the system and to solve them numerically for a scaling cylindrically symmetric flow $[4-5]$.

The problem to be solved consists of different parts, which can be discussed separately: 1. Equation of state, 2. Kinetics of the phase transition, 3. Computation of the flow, 4 . Derivation of the collision terms describing the processing of strangeness, 5. Formulation and solution of the kinetic equations for strangeness evolution. For 1 . we use the bag equation of state for a first order phase transition and for 2 . we assume fast nucleation of the new phase with no supercooling or phase separation. All these are discussed in detail, say, in $[3-5]$. For 3 . we use the flows in [4] computed with the methods in [5]. For 4. we use the collision terms estimated in $[3,6-7]$. The new element in the discussion is the following solution for 5 .

Assume that we know the flow characterised by a certain initial temperature $T_{i}$ and thermalisation time $\tau_{i}$ in a central collision of two equal large nuclei. As the first approximation entropy is conserved in this flow. If mass effects are disregarded in the hadron gas at decoupling, the observed total rapidity density is given by (for $T_{i}>T_{c}$, the transition temperature) 


$$
\frac{d N_{\pi}}{d y}=\frac{4 a}{c} \pi R_{A}^{2} T_{i}^{3} \tau_{i}
$$

where $a=4.6$ (for 2.5 flavours) and $c=3.6$. If also the kaons were in thermal equilibrium, they would, for example, at $T=140 \mathrm{MeV}$ contribute $(K+\bar{K}) /(\pi+K+\bar{K})=0.32$ to the entropy density. Knowing the flow is equivalent to knowing $T(r, r)$ and $v_{r}(\tau, r)$ and during the mixed phase the relative hadron volume fraction

$$
h(\tau, r)=\frac{s_{Q}-s(\tau, r)}{s_{Q}-s_{H}},
$$

where $\tau=\sqrt{t^{2}-z^{2}}, r$ is the radial coordinate and $s_{Q}$ and $s_{H}$ are the entropy densities at $T=T_{c}$,

$$
\frac{s_{Q}}{s_{H}}=r_{d o f}=\frac{42.25}{3}
$$

The kinetic equations describing the evolution of the strangeness density now are as follows. In the initial quark-gluon plasma phase

$$
\begin{aligned}
\partial_{\mu}\left(n_{s} u^{\mu}\right) & =u^{\mu} \partial_{\mu} n_{s}+n_{s} \partial_{\mu} u^{\mu} \\
& =R_{q}(T(\tau, \tau))\left(1-\left(\frac{n_{s}}{n^{e q}}\right)^{2}\right)
\end{aligned}
$$

where

$$
\begin{aligned}
u^{\mu} & =\gamma_{r}\left(\frac{t}{\tau}, v_{r}, 0, \frac{z}{\tau}\right), \quad \gamma_{r}=\frac{1}{\sqrt{1-v_{\tau}^{2}}} \\
n^{e q}(T) & =\frac{g}{2 \pi^{2}} m^{2} T K_{2}\left(\frac{m}{T}\right),
\end{aligned}
$$

$R_{q}(T)$ is the collision term describing the gain and loss caused by the processes $g g \leftrightarrow s \bar{s}$ and $q \bar{q} \leftrightarrow s \bar{s}[6-7], u^{\mu} \partial_{\mu} n_{s}$ is the rate of change of $n_{s}$ in the comoving frame and $n_{s} \partial_{\mu} u^{\mu}$ is the rate of change of $n_{s}$ due to the expansion of the fluid element in the comoving frame. We shall use the following approximation of ref. [3]:

$$
\begin{aligned}
R_{q}(T) & =0.23\left(\frac{T}{m_{s}}\right)^{4} \exp \left(-\frac{2 m_{s}}{T}\right) \frac{1}{f m^{4}} \\
m_{s} & =150 \mathrm{MeV}
\end{aligned}
$$

and the initial condition (for all $r<R_{A}$ ) 


$$
n_{s}\left(\tau_{i}, r\right)=n^{e q}\left(T_{i}\right)
$$

In the mixed phase we have the pair of equations

$$
\begin{aligned}
u^{\mu} \partial_{\mu} n_{s} & =R_{q}\left(T_{c}\right)\left(1-\left(\frac{n_{s}}{n^{e q}}\right)^{2}\right) \\
u^{\mu} \partial_{\mu} n_{K} & =\frac{n_{s}-r_{\text {dof }} n_{K}}{\left(r_{d o f}-1\right) h} \partial_{\mu} u^{\mu}+R_{h}\left(T_{c}\right)\left(1-\left(\frac{n_{K}}{n_{e q}}\right)^{2}\right),
\end{aligned}
$$

where $n_{K}=n_{K^{-}}+n_{\bar{K}^{\circ}}$. Note that the term giving the rate of change of $n_{s}$ due to the expansion of the fluid element in the comoving frame is missing in (7). The collision term $R_{h}$ describes the effect of processes like $K \bar{K} \leftrightarrow n \pi$ and and is only very phenomenologically known [3]. We shall use the simple approximation

$$
R_{h}(T)=0.5 \frac{\sigma}{20 m b}\left(\frac{T}{200 M e V}\right)^{3} \exp \left(-\frac{2 m_{K}}{T}\right) \frac{1}{f m^{4}}
$$

- where $\sigma$ is the pion annihilation cross section and which is a simplified version of the rate term in [3]. The initial condition for (7) at the interface between the quark and mixed phases is

$$
\frac{n_{s}}{n_{K}}=\frac{s_{Q}}{s_{H}}=r_{d o f}
$$

and thus the singularity $h=0$ at the start of the mixed phase is cancelled. Finally, in the hadron phase the kinetic equation is just eq. (4) with $n_{s}$ and $R_{q}$ replaced by $n_{K}$ and $R_{h}$ :

$$
\partial_{\mu}\left(n_{K} u^{\mu}\right)=R_{h}(T(\tau, \tau))\left(1-\left(\frac{n_{K}}{n_{K}^{e q}}\right)^{2}\right)
$$

The equations for the purely longitudinal scaling expansion are simply obtained from the above by the replacements

$$
\begin{aligned}
& u^{\mu} \partial_{\mu} \rightarrow \frac{d}{d t} \\
& \partial_{\mu} u^{\mu} \rightarrow \frac{1}{t}
\end{aligned}
$$

The derivation of the mixed phase kinetic equations is based on the following arguments. Neglect first collisions entirely. Then the total amount of strangeness is conserved and in the mixed phase we have 


$$
\partial_{\mu}\left\{\left[(1-h) n_{s}+h n_{K}\right] u^{\mu}\right\} .
$$

This is one equation for $n_{s}$ and $n_{K}$. To obtain separate equations for $n_{s}$ and $n_{K}$ we must make further assumptions concerning the transmission of strangeness from the quark matter regions (droplets) to the hadron matter regions (bubbles) in the mixed phase. The discussion is actually similar to the discussion of the enrichment of net baryon number in the quark droplets in the quark-hadron phase transition in the early universe [8-9]. We shall make the assumption that the quark-hadron interface is such that it transmits strangeness only in connection with a phase transition through the surface. Under stationary conditions no strangeness would be transmitted and, e.g., the chemical potentials would not be equalised on both sides. The simplest scenario then is obtained by noting that the expansion in the mixed phase does not take place by decreasing $n$ and $T$ but by converting matter from dense quark droplets into less dense hadron bubbles at fixed $T=T_{c}$. Accordingly, the constancy of total strangeness is simplest to satisfy by assuming that strangeness behaves just like entropy density, i.e., eq. (9) is satisfied with constant $n_{s}$ and $n_{K}$ over the entire mixed phase. Thus, without collisions, $u^{\mu} \partial_{\mu} n_{s}=0$. The corresponding equation for $n_{K}$ is obtained by inserting this to (11). This gives the kinetic equations (7) without the collision terms; adding them is straightforward.

The separation of the kinetic equation (11) can also be carried out by assuming that even a stationary quark-hadron interface transmits strangeness and that, accordingly, the chemical potentials on the two sides of the interface can equalize if the transmission is fast enough [10]. The condition $\mu_{K}=\mu_{s}$ is equivalent to

$$
\frac{n_{K}}{n_{a}}=\frac{n_{K}^{e q}}{n_{s}^{e q}}
$$

and the two kinetic equations for the mixed phase are now given by (11) and (12). In [10] the equations are rather formulated in terms of $\mu$ 's than $n$ 's. Since $n_{K}^{\text {eq }} / n_{s}^{\text {eq }}$ is numerically close to $r_{d o f}$, the two sets of equations give very similar results.

The kinetic equations (7) differ from those in [3] in that those in [3] contain the dilution term $-n_{s} / t$ for $n_{s}$ also in the mixed phase, although expansion in the mixed phase does not take place by dilution but by conversion. This leads to a singularity in the equation for $n_{K}$ at the start of the mixed phase, $h=0$.

Next we shall solve the above system of first order partial differential equations in the two variables $\tau$ and $r$ (or ordinary differential equations in $t$ for the no transverse flow $v_{r}=0$ case) and calculate the ratio of the final total strangeness to the final total multiplicity given by the following integral over the decoupling surface with the surface element $d \sigma_{\mu}$ :

$$
\frac{d N_{K} / d y}{d N_{t o t} / d y}=\frac{\int n_{K} u^{\mu} d \sigma_{\mu}}{\int n u^{\mu} d \sigma_{\mu}} .
$$


This is an experimentally observable quantity.

Before giving the numerical results, we shall describe the qualitative behaviour of strangeness during the expansion by working out the solution of the kinetic equations in the $v_{r}=0$ case for the limiting cases $R=0$ and $R=\infty$ (fig. 1). Let us define the total strangeness density (denoted by $n$ without a subscript in the rest of the text)

$$
n=(1-h) n_{s}+h n_{K},
$$

where $h=0$ in the quark phase, $\tau_{i}<\tau<\tau_{Q}, h$ is given by eq. (2) in the mixed phase, $\tau_{Q}<\tau<\tau_{H}=r_{\text {dof }} \tau_{Q}$ and $h=1$ in the hadron phase, $\tau_{H}<\tau$. As we are interested in to what extent strangeness can remain in chemical equilibrium during the expansion, we shall work out how $n / n^{e q}$ behaves.

Initially $n_{u}=n_{d} \approx n_{s}=n_{s}^{e q}\left(T_{i}\right)$ and $n / n^{e q}=1$. If $R_{q}=0, n$ is just diluted by expansion and

$$
n=n_{i} \frac{\tau_{i}}{\tau}=n_{i}\left(\frac{T}{T_{i}}\right)^{3}
$$

The density $n$ is thus not able to decrease as fast as $n^{e q}$ and $n / n^{e q}$ grows. If $R_{q}=\infty, n=$ $n^{e q}$. The ratio between the two outcomes at $T=T_{c}=200 \mathrm{MeV}$ is

$$
r_{s}=\frac{n_{s}\left(m_{s}=0, T_{c}\right)}{n_{s}^{e q}\left(m_{s}, T_{c}\right)}=1.11
$$

(The numbers are for a flow with $T_{i}=500 \mathrm{MeV}, \tau_{i}=0.5 \mathrm{fm}$, corresponding to $d N / d y=$ $26 A$.) Thus, during the quark phase, $n / n^{e q}$ may grow slightly, but the increase is at most about $10 \%$

In the mixed phase we have four cases to study: $\left(R_{q}, R_{h}\right)=(0,0),(\infty, 0),(0, \infty)$ and $(\infty, \infty)$. In the last case clearly $n / n^{e q}=1$. Consider then $\left(R_{q}, R_{h}\right)=(0,0)$. Direct integration of $(7)$ gives

$$
\frac{n}{n^{e q}}=\frac{h / \tau_{\text {dof }}+1-h}{h / r_{e q}+1-h} r_{s}
$$

where

$$
\begin{aligned}
& r_{e q}=\frac{n_{g}^{e q}\left(T_{c}\right)}{n_{K}^{e q}\left(T_{c}\right)} \approx 6.5 \quad\left(T_{c}=200 \mathrm{MeV}\right) \\
& \approx 8.1 \quad\left(T_{c}=160 \mathrm{MeV}\right) .
\end{aligned}
$$


Thus $n / n^{e q}$ decreases during the mixed phase if there are no collisions. Physically this follows from the fact that initially $n_{s} / n_{K}=r_{d o f}$. If there are no collisions, the ratio $n_{s} / n_{K}$ will remain constant and during the mixed phase we have

$$
\frac{n_{K}}{n_{K}^{e q}}=\frac{r_{e q}}{r_{d o f}} \frac{n_{e}}{n_{s}^{e q}} .
$$

The ratio $n / n^{e q}$ then decreases from the initial value $r_{B}=n_{a} / n_{g}^{e q}$ to the final value $r_{a}\left(r_{e q} / r_{d o f}\right)=n_{K} / n_{K}^{e q}$. The other two pairs of values of $R_{q}, R_{h}$ are worked out similarly (fig. 1)

Finally, if $R_{h}=0$ in the hadron phase, $n / n^{e q}$ again starts increasing. This happens since $n_{K}^{\text {eq }}$ decreases more rapidly with $T$ because of mass suppression than $n_{K}$, which decreases only due to dilution. How far $n / n^{e q}$ will have time to increase depends on the details of the decoupling process. From fig. 1 one might think that the final prediction is very uncertain. This is so for $n / n^{e q}$, but the crucial number is actually $n / s$, which, in practice, is remarkably insensitive to the decoupling temperature (see fig. $3 \mathrm{~b}$ ). The reason for this is that, in realistic cases, $R_{h}$ is large in the mixed phase but decreases very rapidly upon entering the hadron phase. In the hadron phase little happens to strangeness, both $n$ and $s$ are just diluted and $n / s$ is constant.

The overall conclusion thus is that the final $n_{K}$ ( $=n_{K}$ just in the beginning of the hadron phase) will be near or at most a factor 0.5 times the equilibrium value. In thermal equilibrium we would have $\left(g_{K}\right.$ counts the number of kaon types in $n_{K}$; earlier we defined $n_{K}=n_{K^{-}}+n_{\bar{K}^{\circ}}$, similarly $\left.g_{\pi}\right)$

$$
\begin{aligned}
\frac{n_{K}}{n_{\pi}}=\frac{g_{K}}{g_{\pi}} 0.42 & (T=200 \mathrm{MeV}) \\
0.30 & (T=160 \mathrm{MeV}) \\
0.24 & (T=140 \mathrm{MeV}) .
\end{aligned}
$$

The expected ratio $N_{K^{-}} / N_{\pi^{-}}$is thus given by $0.5 \ldots 1$ times $0.42 \ldots . .24$, depending on the decoupling temperature. Equivalently, the expected ratio $\left(N_{K}+N_{\bar{K}}\right) /\left(N_{\pi}+N_{K}+N_{\bar{K}}\right)$, summing over charges, would vary between $0.34\left(T_{\text {dec }}=200\right)$ and $0.24\left(T_{\text {dec }}=140\right)$ to something less by a factor 2 . There is a slight increase relative to the initial $n_{s} / n_{\text {tot }}=0.10$, but not a large one.

Numerical results for the evolution of the strangeness density for the case with transverse expansion are shown in figs. 2-3. For brevity, results are only presented for the flow with $T_{i}=500 \mathrm{MeV}, \tau_{i}=0.5 \mathrm{fm}$ (corresponding to $d N / d y=26 \mathrm{~A}$ for massless particles) and a first-order phase transition with $T_{c}=200 \mathrm{MeV}$ and $r_{\text {dof }}=14$ (fig. 2). If the equation of state is not of first order but a continuous one with as large a jump in entropy density $\left(s_{Q} / s_{H}=14\right)$, as indicated by lattice Monte Carlo calculations [11], the overall pattern of the flow should be rather similar, only the large space-time region of constant 
$T=T_{c}=200 \mathrm{MeV}$ would be replaced by an equally large region in which $T$ varies slowly around $200 \mathrm{MeV}$.

Figs. $3 \mathrm{a}$ and $3 \mathrm{~b}$ show the ratios $n / n^{e q}$ (eq. (14)) and $3.6 n / \mathrm{s}$ (3.6 is the appropriate factor for massless particles to convert $s$ to multiplicity) with the rate terms as given in eqs. (5) and (8). Fig. $3 c$ shows $n / n^{e q}$ if the rate term in hadron phase is reduced by a factor 10 [11]. Here $n^{e q}$ and $s$ are calculated directly from the flow; $n$ is obtained by numerically integrating the kinetic equations (4-10).

To obtain a number directly comparable with experiment, the flux across the decoupling surface is calculated according to eq. (13). The numerical value of $N_{K} /\left(N_{\pi}+N_{K}+N_{K}\right)$ (summing over charges) corresponding to $T_{d e c}=140 \mathrm{MeV}$ is, as can be estimated from figs. $3 \mathrm{~b}$ and $3 \mathrm{c}, 0.15$ for the full hadronic rate term $R_{h}$ and 0.11 for the reduced term.

These numerical results are in agreement with the qualitative discussion above (fig. 1) and the estimates given on the basis of eq. (18). Because $r_{e q} / r_{d o f}<1$, the strangeness density falls below the equilibrium density in the mixed phase and remains the lower the smaller $R_{h}$ is. In hadron phase a rapid increase of $n / n_{e q}$ is seen. This happens because the $K \bar{K}$ reactions effectively freeze out due to the fast transverse expansion in the hadron phase leading to a situation where $n$ decreases only because of the increase in volume, whereas $n^{\text {eq }}$ decreases also due to the mass effect. As a consequence of the above behaviour of $n$, $n / s$ is essentially constant in the hadron phase and thus, in particular, the choice of the decoupling temperature does not matter.

The transverse flow affects the evolution of strangeness mainly by reducing the time scales in the problem. The quark phase is essentially unaffected. For the flow in fig. 2 the mixed phase in the $v_{r}=0$ case would last for $7.8 \mathrm{fm}<\tau<110 \mathrm{fm}$. The transverse flow reduces this timescale to less than $40 \mathrm{fm}$, which is essentially the same as the equilibration time scale (3). This leads to the factor of 2 uncertainty in the value of $n / n^{e q}$ at the end of the mixud phase. The largest reduction in the time scales is in the lifetime of the hadron phase. Together with the rapid drop in densities, caused by the shock at the mixed phasehadron phase interface, this means that the transverse flow effectively cuts off the hadron phase from the processing of strangeness.

Finally, with the calculated $n_{K}$ we may also discuss the predicted transverse momenta of kaons and, in particular, the mass effect. This question has earlier been discussed by assuming that $n_{K}=n_{K}^{e q}\left(T_{c}\right)$ along the decoupling curve [12]. The inspection of contour curves in figs. 3 shows, that on a large fraction of the decoupling curve, which for $T_{\text {dec }}=$ $140 \mathrm{MeV}$ is shown as the dashed-dotted line, $n_{K}$ varies very little. This means, that the decoupling integral for the kaon multiplicity satisfies

$$
\frac{d N_{K}}{d y}=\int n_{K} u^{\mu} d \sigma_{\mu} \approx \frac{n_{K}}{n_{K}^{e q}} \int n_{K}^{e q} u^{\mu} d \sigma_{\mu},
$$

where $n_{K} / n_{K}^{e q}$ denotes the roughly constant value of this ratio on the main part of the 
decoupling curve. Similarly, only the normalization of the decoupling integral for the $p_{T}$-distribution $[5,12]$ may change appreciably, but the shape and the resulting average value of transverse momentum, $\left\langle p_{T}\right\rangle$, remain almost the same when $n_{K}$ instead of $n_{K}^{\text {eq }}$ is used. The actual numerical results show a small decrease of $\left\langle p_{T}\right\rangle$ (from $1.040 \mathrm{GeV}$ for $n_{K}^{\text {eq }}$ to $0.996 \mathrm{GeV}$ for $n_{K}$ with full $R_{h}$ ) and a sharpening of the $p_{T}$-distribution. This can be understood to result from the fact that the region around the collision axis, where transverse velocity is small is, due to the longer timescale, relatively richer in strangeness $\left(n_{K}>n_{K}^{e q}\right)$ than the outer regions which flow fast and decouple so rapidly that $n_{K}$ does not reach the equilibrium value.

Summarising, we have derived kinetic equations for the evolution of strangeness in the central region of an ultra-relativistic nuclear collision and solved them numerically for a longitudinally scaling and tranversally cylindrically symmetric expansion. The conclusion is that the strangeness density evolves close to or at most a factor 2 below the equilibrium density at the end of the mixed phase. In hadron phase the rapid increase of the volume due to the transverse flow leads to a rapid freeze out of the strangeness annihilation reactions, making the final amount of strangeness rather independent of the other properties of the hadron phase. Depending on the details of the rates of the strangeness reactions and time scales of the flow, the final $K / \pi$ ratios will be $1 . .3$ times those observed in $N \cdot N$ collisions. This conclusion is the same as that obtained with kinetic equations [11] based on slightly different assumptions of the transport of strangeness through the quark-hadron interface. The shape of the $p_{T}$-distribution and the average value of the transverse momentum of kaons are insensitive to the rate of strangeness reactions and the total amount of kaons.

Acknowledgements: We thank J. Kapusta, T. Matsui and L. McLerran for discussions and correspondence. 


\section{References}

1. For a review, see B. Müller, The Physics of the Quark-Gluon Plasma, Lecture Notes in Physics 225, Springer Verlag, Heidelberg, (1985)

2. M. Gyulassy, Nucl. Phys. A418, (1984) 59c

3. J. Kapusta and A. Mekjian, Phys. Rev. D33 (1986) 1304.

4. K. Kajantie, M. Kataja, L. McLerran and P.V. Ruuskanen, Helsinki preprint HUTFT-86-6, (1986), submitted to Phys.Rev.D.

5. E. von Gersdorff, M. Kataja, L. McLerran and P.V. Ruuskanen, Fermilab preprint $86 / 13-\mathrm{T},(1986)$.

6. J. Rafelski and B. Müller, Phys. Rev. Lett. 48 (1982) 1066.

7. T. Matsui, B. Svetitsky and L. McLerran, MIT preprint CTP 1320, (1985).

8. E. Witten, Phys. Rev. D30 (1984) 272.

9. K. Kajantie and H. Kurki-Suonio, Univ. of Texas, Austin, Preprint,(1986).

10. T. Matsui, B. Svetitsky and L. McLerran, MIT Preprint CTP 1344, (1986).

11. R. Gavai and F. Karsch, Nucl. Phys. B261 (1985) 273.

12. M. Kataja, P.V. Ruuskanen, L. McLerran and E. von Gersdorff, Fermilab preprint 86/73-T,(1986). 
Figure captions

Fig. 1 Qualitative behavious of the total strangeness density $n$ (eq. (14)) relative to its equilibrium value $\pi^{e q}$ for possible combinations of vanishing or infinite strangeness equilibration rates $R_{q}$ and $R_{h}$ in the quark and hadron phases for the case of no transverse velocity. The quantities $\tau_{d o f}, r_{e q}, r_{A}, \tau_{Q}$ and $r_{H}$ are defined in the text. In realistic cases, $R_{h}$ is large in the mixed phase and decreases rapidly after entering the hadron phase. Thus the final value of $n / n^{2 q}$ will lie between the $R_{h}=0$ curves. To predict experimentally measurable quantities, $n / s$ is needed and this ratio is constant in the hadron phase.

Fig. 2 Curves of constant energy density in units of $\mathrm{GeV} / \mathrm{fm}^{3}$ for a flow with $T_{i}=500 \mathrm{MeV}$, $\tau_{i}=0.5 \mathrm{fm}$ and a first order phase transition with $T_{c}=200 \mathrm{MeV}$ and $\tau_{d o f}=14$ [4] plotted in the proper time (here $t$ ) vs radial coordinate $r$ plane. The mixed phase lies between the thicker curves with $\epsilon=\epsilon_{Q}=3.6$ and $\epsilon=\epsilon_{H}=0.20$. The dashed-dotted curve corsesponds to $T=140 \mathrm{MeV}$, an approximation to the decoupling temperature.

Fig. 3a The ratio $n / n^{e q}$ ( $n$ is the total strangeness density) for the flow in fig. 2 and with the rate terms as given in the text. The lines for $\epsilon_{Q}, \epsilon_{H}$ and decoupling temperature are as in fig. 2. The lines close to the light cone are an artifact of the graphics.

$3 b$ The ratio $n /(3.6 s)$ for the flow in fig. 2 and with the rate terms as given in the text.

3c. The ratio $r i / \pi^{e q}$ for the flow in fig. 2 and with the hadron phase rate term in eq. (8) reduced by a factor 10 .

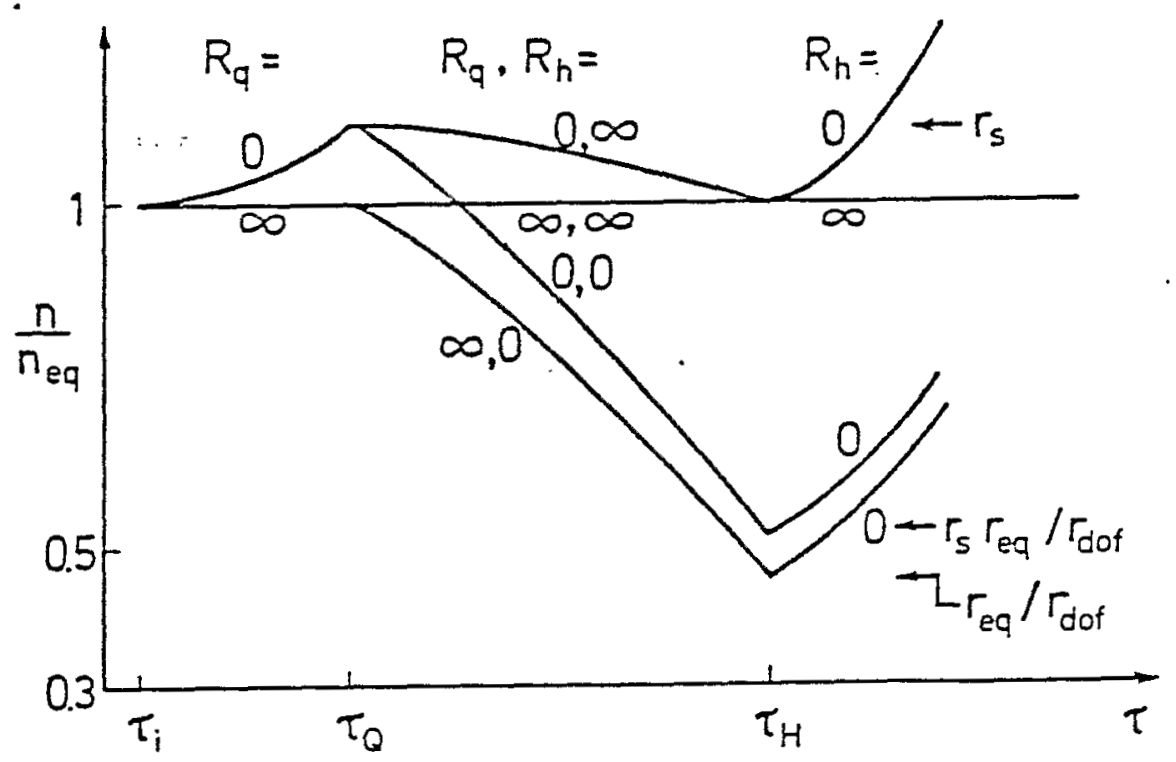

Fig.1 
ENERGY DENSITY
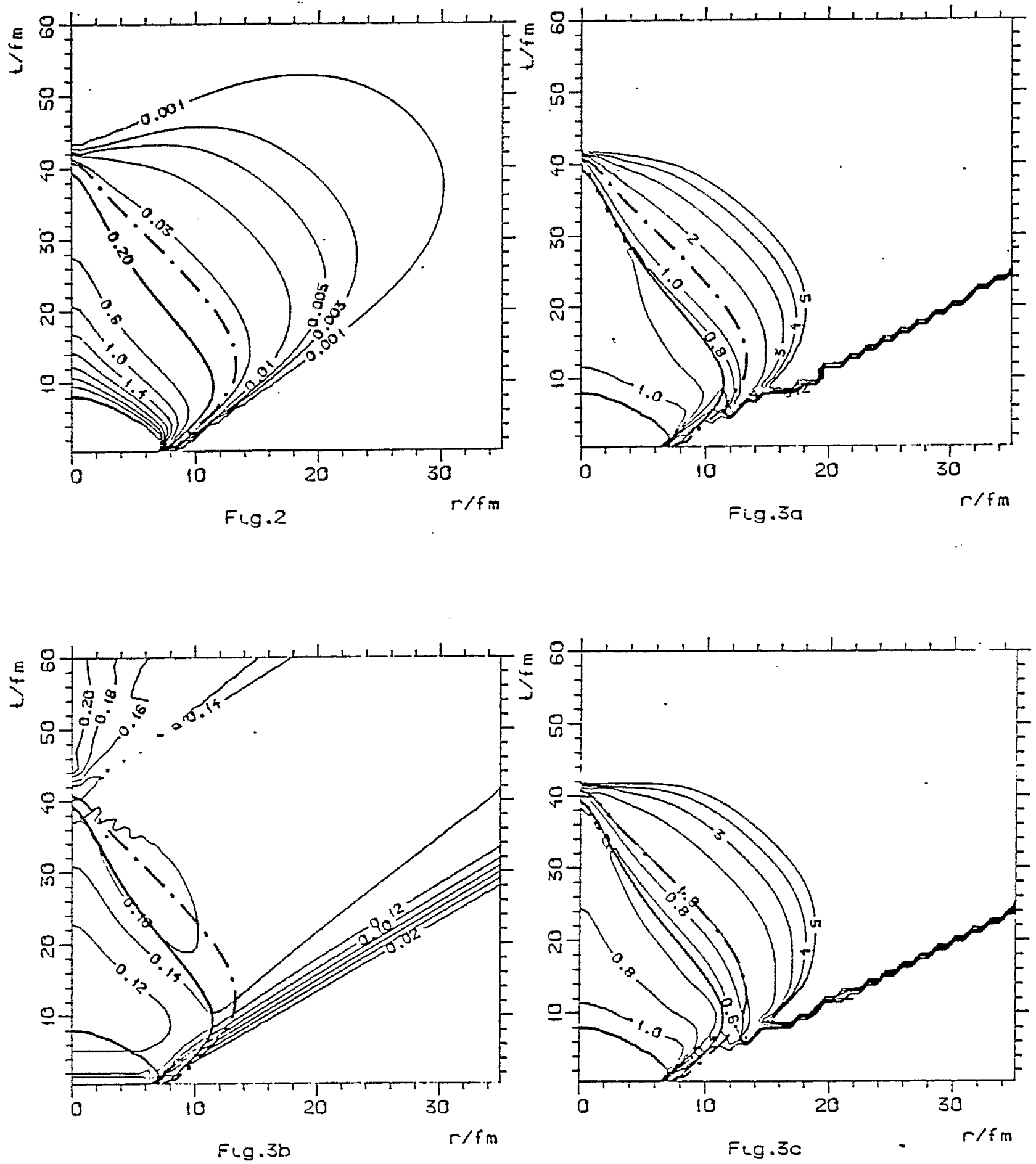